05,07

\title{
Управление магнитной структурой CoNi-микрочастиц при помощи механических напряжений
}

\author{
() Н.И. Нургазизов, Д.А. Бизяев, А.А. Бухараев, А.П. Чукланов \\ Казанский фризико-технический институт им. Е.К. Завойского, \\ Федеральный исследовательский центр „Казанский научный центр РАН“, \\ Казань, Россия \\ E-mail: niazn@mail.ru
}

Поступила в Редакцию 26 марта 2020 г.

В окончательной редакции 26 марта 2020 г.

Принята к публикации 2 апреля 2020 г.

\begin{abstract}
Исследовалось изменение доменной структуры CoNi-микрочастиц под действием механических напряжений. Для этого на поверхности полированной стеклянной подложки был сформирован массив одинаковых планарных CoNi-частиц квадратной формы. Для создания механических напряжений в частицах использовался упругий изгиб подложки. Методами магнитно-силовой микроскопии было показано, что при помощи механических напряжений можно изменять магнитную структуру частиц от мультидоменной до квазиоднородного намагничивания.
\end{abstract}

Ключевые слова: планарные магнитные микрочастицы, магнитно-силовая микроскопия, магнитоупругий эффект.

DOI: 10.21883/FTT.2020.09.49777.16H

\section{1. Введение}

Магнитоупругий эффект (эффект Виллари) или влияние механических напряжений на магнитные свойства ферромагнитных материалов можно использовать для управления намагниченностью микро- и наночастиц [1-4]. Интерес к такому способу управления намагниченностью связан с тем, что при таком подходе можно существенно снизить общие затраты энергии, необходимой для изменения направления намагниченности однодоменной частицы. На основе таких частиц можно создавать низкоэнергетические устройства для обработки и хранения информации $[2,3]$. Для изучения магнитной структуры и процесса переключения намагниченности отдельно взятой частицы или их небольшого массива одним из наиболее перспективных методов является магнитно-силовая микроскопия. С помощью магнитно-силового микроскопа (МСМ) можно проводить исследования магнитной структуры при различных внешних условиях с высоким пространственным разрешением (до $10 \mathrm{~nm}$ ). В ряде случаев, за счет использования микрочастиц определенной формы, можно также решить и обратную задачу по определению механических напряжений, действующих на частицу, на основе анализа ее магнитной структуры $[4,5]$. Для визуализации механических напряжений в приповерхностном слое можно использовать не только частицы, но и сплошные пленки $[6,7]$, но для изучения магнитной структуры пленки не всегда можно применять МСМ-методы.

В настоящей работе исследовалось влияние механического напряжения на магнитную структуру планарных CoNi-микрочастиц, имеющих квадратную форму. Ранее, на примере пермаллоевых (Ру) микрочастиц, было показано, что частицы квадратной формы можно использовать для определения направления одноосного механического напряжения и для оценки их величины. Однако для изменения магнитной структуры Ру частиц с латеральными размерами $7.5 \mu \mathrm{m}$ требовались очень большие механические напряжения, которые было невозможно реализовать экспериментально из-за разрушения образца. Поэтому для данного исследования частицы были изготовлены из CoNi-сплава, который имеет гораздо более высокую константу магнитострикции насыщения $\lambda_{s}=-25 \cdot 10^{-6}$, по сравнению с Ру $-\lambda_{s}=-2 \cdot 10^{-6}[5]$. Это должно было, по нашим предположениям, увеличить чувствительность магнитной структуры частицы к механическому напряжению и позволить эффективно управлять ею при помощи напряжения.

\section{2. Подготовка образцов и методика проведения измерений}

Для проведения исследований были изготовлены два типа образцов с частицами размером $25 \times 25 \times 0.03 \mu \mathrm{m}$ и $7.5 \times 7.5 \times 0.03 \mu \mathrm{m}$. Массив одинаковых частиц был сформирован на поверхности полированной стеклянной подложки толщиной $0.15 \mathrm{~mm}$. Для напыления частиц использовался метод испарения электронным лучом твердотельной мишени из CoNi-сплава (Co18\%, Ni82\%) в сверхвысоком вакууме. Напыление проводилось через металлическую сетку с одинаковыми квадратными отверстиями, которая была плотно прижата к поверхности подложки. Латеральные размеры использованной стеклянной подложки составляли $18 \mathrm{~mm}$. После изготов- 


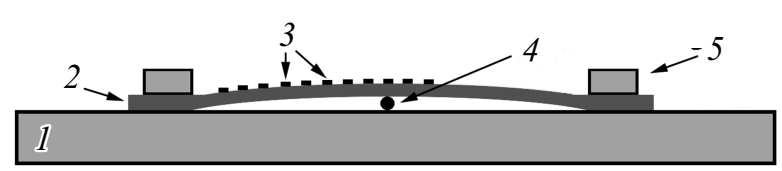

Рис. 1. Схема изгиба образца при проведении исследований. Цифрами обозначено: 1 - держатель образца, 2 - стеклянная подложка, 3 - сформированные на ней частицы, $4-$ проволока для изгиба подложки, 5 - зажим для подложки.

ления образец с частицами выдерживался в условиях вакуума при температуре $300^{\circ} \mathrm{C}$ в течение $15 \mathrm{~min}$, что позволяло отжечь большую часть дефектов, образующихся при напылении.

Для исследования CoNi-частиц использовалась установка Solver HV (NT MDT) работающая в MCM-режиме или в режиме атомно-силового микросокпа (АCM). В качестве МСМ-сигнала регистрировалась разность фаз между сигналом с фотодиода, регистрирующего реальные колебания кантиливера, и сигналом, возбуждающим эти резонансные колебания. МСМ-измерения проводились по однопроходной методике, когда при сканировании образца зонд перемещается на постоянной высоте над поверхностью исследуемого образца. В этом случае МСМ-зонд не искажает распределение намагниченности в CoNi-частицах. Для проведения исследований использовались кантиливеры „Multi75M-G“ (BudgetSensor) и „PPP-LM-MFMR“ (Nanosensors).

Для создания напряжений в частицах подложка упруго изгибалась за счет крепления в специальном держателе (рис. 1). Держатель представлял собой ровную пластину, изготовленную из немагнитного материала. Под центр исследуемого образца перпендикулярно его длинной оси подкладывалась металлическая проволока, а края образца плотно прижимались при помощи зажимов к поверхности держателя. За счет этого происходил неравномерный изгиб подложки, и деформация подложки приводила к деформации расположенных на ней частиц. Как показали проведенные нами [5] и другими авторами оценки [6], в частицах, расположенных вдоль длинной центральной оси подложки, создается одноосное напряжение, направление которого совпадает с этой осью, а величина изменяется при удалении от центра проволоки. Для используемого нами держателя применение проволоки диаметром $50 \mu \mathrm{m}$ позволяет создавать в частицах, расположенных на подложке непосредственно над проволокой напряжение $0.08 \mathrm{GPa}$. В первом приближении напряжение линейно уменьшается до нуля при приближении к зажимам. Проволока диаметром $135 \mu \mathrm{m}$ позволяет создавать напряжения до $0.24 \mathrm{GPa}$ в области над проволокой. Таким образом, подбирая нужный диаметр проволоки, можно изучать различные диапазоны механических напряжений. При этом создаваемые напряжения симметричны относительно центра проволоки, что позволяет изучать его распределение только на одной половине образца.

\section{3. Доменная структура CoNi-частиц}

Топографическое изображение отдельной $\mathrm{CoNi}$-частицы с размерами $7.5 \times 7.5 \times 0.03 \mu \mathrm{m}(7.5 \mu \mathrm{m})$ приведено на рис. 2, a. Аналогичное изображение для CoNi-частицы с размерами $25 \times 25 \times 0.03 \mu \mathrm{m}(25 \mu \mathrm{m})$ представлено на рис. 3,a. Изображения, полученные в разных местах образца, позволили подтвердить, что все частицы на образце имеют одинаковые размеры и форму. Это позволяет сравнивать магнитные изображения от частиц, полученные в разных областях образца друг с другом.

МСМ-изображение, характерное для $7.5 \mu \mathrm{m}$ частиц при отсутствии механического напряжения, приведено на рис. $2, b$. Для того чтобы определить распределение намагниченности в частице, полученное МСМ-изображение сравнивалось с результатом компьютерного моделирования ее магнитной структуры. Данные расчеты (полученные распределения схематично представлены под каждым МСМ-изображением на рис. 2,3) были выполнены при помощи программы OOMMF [8]. Намагниченность насыщения используемого сплава $\mathrm{CoNi}$ принималась за $650 \mathrm{kA} / \mathrm{m}$, константа обменного взаимодействия - $1.1 \cdot 10^{-11} \mathrm{~J} / \mathrm{m}$ [9]. При моделировании частица разбивалась на элементарные ячейки с размерами в плоскости частицы $20 \mathrm{~nm}$ и в направлении нормали к плоскости частицы $30 \mathrm{~nm}$ (что равно высоте частицы). Затем, за счет изменения направления намагниченности, в каждой ячейке минимизировалась общая магнитная энергия частицы. Полученное распределение намагниченности использовалось для моделирования МСМ-изображения при помощи программы „Virtual MFM“ [10], которое сравнивалось с экспериментальными результатами. Совпадение полученного модельного изображения с экспериментальным позволяло сделать вывод о соответствии этого расчета реальному распределению намагниченности.

Для того чтобы добиться совпадения виртуальных МСМ-изображений с экспериментальными (т.е. найти распределение намагниченности в частице), когда на частицу действует механическое напряжение, при проведении ООММF-вычислений варьировалось значение коэффициента анизотропии $\left(K_{\mathrm{eff}}\right)$. В рассматриваемом нами случае эффективный коэффициент анизотропии частицы зависит от константы магнитокристаллической анизотропии и от коэффициента магнитоупругой анизотропии. Варьирование $K_{\text {eff }}$ при моделировании распределения намагниченности в микрочастицах в зависимости от механического напряжения часто используется и другими авторами, например, в работе [4] для исследования никелевых частиц. Исследуемые $\mathrm{CoNi}$-частицы за счет своего состава имели константу магнитокристаллической анизотропии близкой к 0 и поликристаллическую структуру, поэтому эффективный коэффициент анизотропии частицы определяется коэффициентом магнитоупругой анизотропии.

Согласно полученным МСМ-изображениям для $7.5 \mu \mathrm{m}$ частиц, при отсутствии механического напряже- 

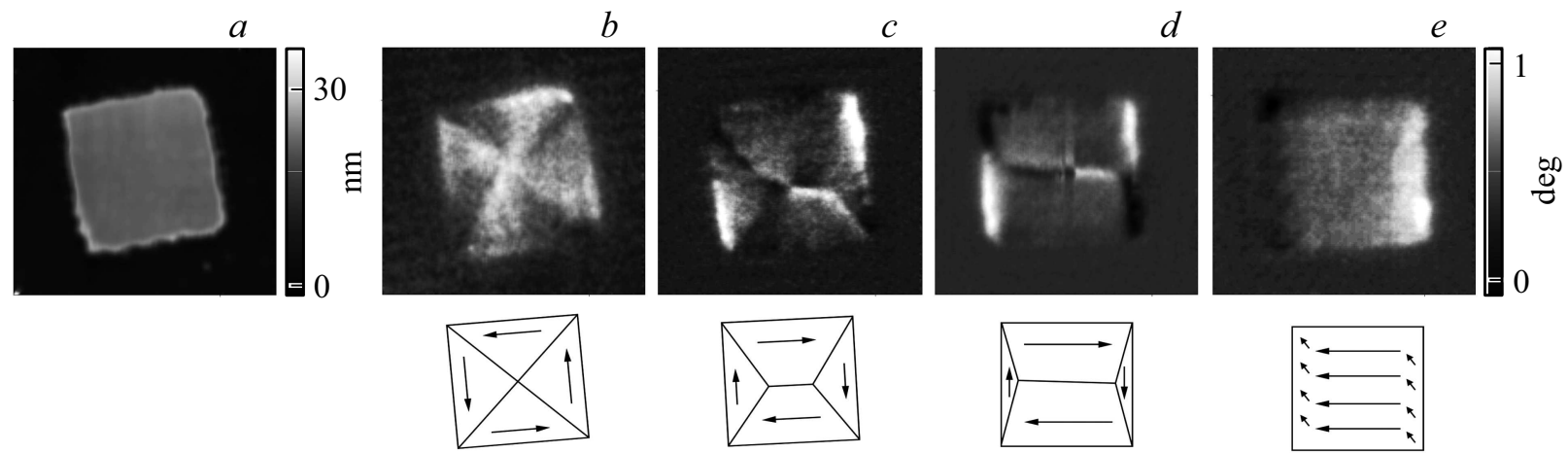

Рис. 2. Топографическое изображение $7.5 \mu \mathrm{m}$ CoNi-частицы $(a)$. MCM-изображения CoNi-частицы: в четырехдоменном состоянии при отсутствии механических напряжений $(b)$, в четырехдоменном состоянии при растяжении $0.1 \mathrm{GPa}(c)$, при растяжении $0.2 \mathrm{GPa}(d)$, в квазиоднородном состоянии при растяжении свыше $0.2 \mathrm{GPa}(e)$. Снизу приведены соответствуюшее МСМ-изображению распределение намагниченности. Растяжение направлено вдоль оси $Y$. Размер поля сканирования $12.5 \times 12.5 \mu \mathrm{m}^{2}$.
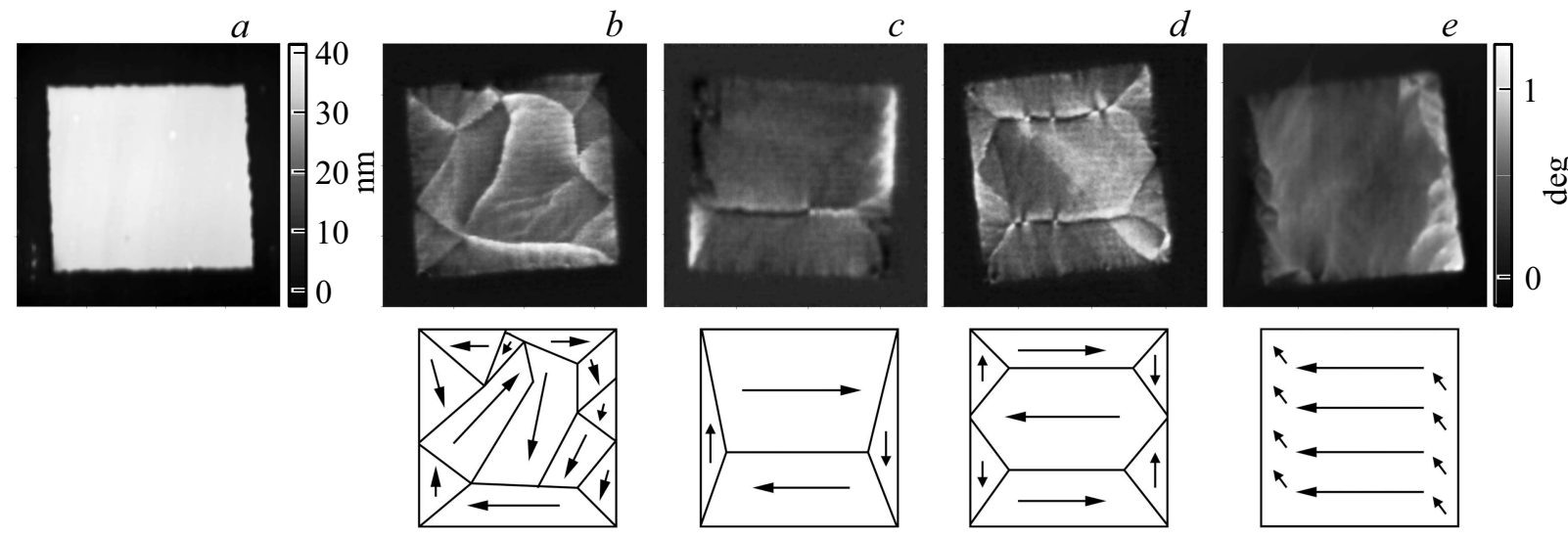

Рис. 3. Топографическое изображение $25 \mu \mathrm{m}$ CoNi-частицы (a). МСМ-изображения $25 \mu \mathrm{m}$ СоNi-частиц: в мультидоменном состоянии при отсутствии механических напряжений $(b)$, в четырехдоменном состоянии при растяжении $0.03 \mathrm{GPa}(c)$, в семидоменном состоянии при растяжении $0.05 \mathrm{GPa}(d)$, в квазиоднородном состоянии при растяжении свыше $0.1 \mathrm{GPa}(e)$. Снизу приведены соответствующие МС-изображениям распределения намагниченности. Напряжение прикладывается вдоль оси $Y$. Размер поля сканирования $35 \times 35 \mu \mathrm{m}^{2}$.

ния, характерной является четырехдоменная структура (рис. $2, b$ ). При этом все домены имеют приблизительно одинаковый размер и форму. Направление намагниченности в них параллельно стороне частицы, у которой расположен домен. Небольшие искажения, наблюдаемые на отдельных экспериментальных МСМ-изображениях частиц, скорее всего обусловлены локальными дефектами, которые не воспроизводятся при OOMMFмоделировании.

МСМ-изображение, характерное для $25 \mu \mathrm{m}$ частиц при отсутствии механического напряжения, приведено на рис. $3, b$ и оно соответствует мультидоменному состоянию. Такое состояние носит довольно случайный характер и сильно зависит от локальных дефектов, индивидуальных для каждой конкретной частицы. Поэтому рассчитать с помощью ООММF распределение намагниченности, точно соответствующее полученному МСМ-изображению, не представляется возможным, и на схеме (рис. $3, b)$ приводится возможное распреде- ление намагниченности, которое может не совпадать с реальным.

Для создания механических напряжений, влияющих на магнитную структуру частиц, образец размещался в специальном держателе (рис. 1). За счет неравномерного упругого изгиба подложки в держателе можно было, перемещая поле сканирования МСМ-зонда по образцу, изучать влияние различных по величине механических напряжений на магнитную структуру частиц. При растяжении $7.5 \mu \mathrm{m}$ частиц за счет магнитоупругого эффекта происходило увеличение размера доменов, намагниченность которых была перпендикулярна направлению растяжения (рис. 2,c). Между двумя этими доменами формируется доменная стенка, которая на МСМ-изображении наблюдается как характерная перемычка. Длина этой перемычки пропорциональна соотношению между площадями доменов с направлением намагниченности параллельной и перпендикулярной оси растяжения частицы (рис. $1, c, d)$ и может быть использована для 
определения величины механического напряжения, действующего на частицу.

В интервале от 0.05 до $0.15 \mathrm{GPa}$ можно было изменять размеры доменов за счет изменения напряжения, действующего на частицу (рис. $1, c$ ). При более высоких напряжениях магнитная структура частицы практически не менялась, а при напряжениях свыше $0.2 \mathrm{GPa}$ частица могла перейти в состояние квазиоднородной намагниченности (рис. 1,e). Напряжения свыше $0.2 \mathrm{GPa}$ в CoNi-частицах были трудно достижимы, т. к. они приводили к разрушению подложки. Таким образом, механические напряжения позволяют управлять размерами доменов планарных $7.5 \mu \mathrm{m} \mathrm{CoNi-частиц,} \mathrm{находящихся} \mathrm{в}$ четырехдоменном состоянии. А плавное увеличение размера доменов при увеличении механического напряжения позволяет эффективно использовать такие частицы для определения величины этого напряжения.

У $25 \mu \mathrm{m}$ CoNi-частиц наблюдалась большая чувствительность магнитной структуры к механическим напряжениям. При значении механического напряжения больше $0.01 \mathrm{GPa}$ происходило изменение магнитной структуры с мультидоменной на четырехдоменную (рис. 3, c) или семидоменную (рис. 3,d). При этом относительно небольшое растяжение приводило к существенному увеличению размера доменов в перпендикулярном направлении, и оно слабо зависело от величины приложенного напряжения. Как и в случае с $7.5 \mu \mathrm{m}$ частицами увеличение размеров перпендикулярных доменов связано с отрицательным знаком константы магнитострикции используемого материала. При значении механического напряжения свыше $0.1 \mathrm{GPa}$ частицы становились квазиоднородно намагниченными (рис. 3,e). Таким образом у $25 \mu \mathrm{m}$ CoNi-частиц механическими напряжениями можно было только эффективно переключать магнитную структуру из мультидоменного состояния в четырехили семидоменное и в квазиоднородное. Это позволяет использовать такие частицы только для качественного детектирования механического напряжения, без количественной оценки его величины.

\section{4. Заключение}

Методами магнитно-силовой микроскопии было показано, что механические напряжения позволяют изменять магнитную структуру CoNi-частиц. В случае с планарными частицами размером $7.5 \mu \mathrm{m}$, варьируя величину действующего на них механического напряжения, можно изменять размеры магнитных доменов. В случае $25 \mu \mathrm{m}$ частиц с помощью механических напряжений можно переключать ее между мультидоменным и квазиоднородно намагниченным состояниями. Такие частицы можно использовать для детектирования наличия механических напряжений, т. к. переход из мультидоменного состояния в четырех- или семидоменное происходит при низких значениях действующего на частицу механического напряжения. В то же время $7.5 \mu \mathrm{m}$ частицы могут быть использованы для определения величины действующего на них напряжения, т. к. их четырехдоменная структура может сохраняться вплоть до значений напряжения в $0.2 \mathrm{GPa}$.

\section{Финансирование работы}

Работа была выполнена в рамках Государственного задания Федерального исследовательского центра „Казанский научный центр РАН““. МСМ-исследования были проведены при поддержке РФФИ (проект 18-02-00204).

\section{Конфликт интересов}

Авторы заявляют, что у них нет конфликта интересов.

\section{Список литературы}

[1] А.И. Морозов. ФТТ 56, 833 (2014).

[2] А.А. Бухараев, А.К. Звездин, А.П. Пятаков, Ю.К. Фетисов. УФН 188, 1288 (2018).

[3] D.E. Nikonov, I.A. Young. J. Mater. Res. 29, 2109 (2014).

[4] S. Finizio, M. Foerster, M. Buzzi, B. Kruger, M. Jourdan, C. Vaz, J. Hockel, T. Miyawaki, A. Tkach, S. Valencia, F. Kronast, G. Carman, F. Nolting, M. Klaui. Phys. Rev. Appl. 1, 021001 (2014).

[5] Н.И. Нургазизов, Д.А. Бизяев, А.А. Бухараев, И.В. Русских, Ю.В. Садчиков. ЖТФ 89, 1663 (2019).

[6] Б.А. Беляев, А.В. Изотов. ФТТ 49, 1651 (2007).

[7] D. Cao, Z. Wang, L. Pan. Appl. Phys. A 122, 938 (2016).

[8] M.J. Donahue, D.G. Porter. OOMMF User's Guide, Version 1.0. National Institute of Standards and Technology, Gaithersburg, MD, USA (1999).

83 p. http://math.nist.gov/oommf.

[9] P. Talagala, P.S. Fodor, D. Haddad, R. Naik, L. E. Wenger, P.P. Vaishnava, V.M. Naik. Phys. Rev. B 66, 144426 (2002).

[10] Д.В. Овчинников, А.А. Бухараев. ЖТФ 71, 8, 85 (2001).

Редактор Т.Н. Василевская 